\title{
Exact procedures for the analysis of multidimensional contingency tables
}

\author{
JULIET POPPER SHAFFER \\ University of Kansas, Lawrence, Kansas 66044
}

The log-linear model for contingency tables expresses the logarithm of a cell frequency as an additive function of main effects, interactions, etc., in a way formally identical with an analysis of variance model. Exact statistical tests are developed to test hypotheses that specific effects or sets of effects are zero, yielding procedures for exploring relationships among qualitative variables which are suitable for small samples. The tests are analogous to Fisher's exact test for a $2 \times 2$ contingency table. Given a hypothesis, the exact probability of the obtained table is determined, conditional on fixed marginals or other functions of the cell frequencies. The sum of the probabilities of the obtained table and of all less probable ones is the exact probability to be considered in testing the null hypothesis. Procedures for obtaining exact probabilities are explained in detail, with examples given.

A multidimensional contingency table results when each observation in a sample is classified according to its value on two or more categorical variables. When there are two variables, the definition of independence, or lack of association, between them is relatively straightforward. The familiar chi-square test can be used as an approximate test of the hypothesis of independence. Fisher's exact test is an exact procedure for a $2 \times 2$ table; the analogous exact procedure for larger tables is a special case of the general method presented in this paper. When there are more than two variables, the definitions of relationships of varying degrees of complexity are not obvious, and different approaches have been taken. In a previous paper (Shaffer, 1972), it was argued that the loglinear model provides a framework suitable for a general-purpose analysis of the relationships in contingency tables. The properties of the model and reasons for its choice were discussed in detail, and it was pointed out that it had been advocated by many statisticians from a variety of perspectives.

Considerable effort has gone into the development of approximate statistical tests of hypotheses within the framework of the model. One type of approximate test, the type most similar to the standard method in two-factor tables, requires the estimation of expected cell frequencies under a given hypothesis and the computation of an ordinary chi-square statistic. This test procedure was used in a later part of the present paper where the outcomes of the exact and approximate methods are compared. Aside from the requirement of adequate sample size, such tests are not always practical, because under some circumstances the computation of expected cell frequencies is extremely complex. For a discussion and comparison of various approximate procedures and an extensive bibliography, see Shaffer (1972).

Relatively little attention has been paid to exact tests, valid regardless of sample size. Bartlett (1935), in the first paper on multidimensional contingency table analysis, presented an exact test for three-factor interaction in a $2 \times 2 \times 2\left(2^{3}\right)$ table, and Freeman and Halton (1951) presented exact methods for testing some hypotheses of interest, useful suggestions for simplifying calculations in all such tests, and a general overview of the theoretical rationale underlying them. The purpose of this paper is to present a comprehensive set of exact methods, for general multidimensional contingency tables, for testing hypotheses of the type normally tested in an overall analysis of variance.

\section{NOTATION}

The variables in the contingency table will be denoted $A$, $B, C, \cdots$, with a particular category of $A$ denoted $A_{i}, i=1$, $\cdots$, a; of $B$ denoted $B_{j}, j=1, \cdots$, b; etc. Further notation will be defined in the context of a three-factor table. The probability that an entity appears in the cell characterized by level $i$ of $A, j$ of $B$, and $k$ of $C$ will be denoted $p_{i j k}$, with

$$
\underset{\text { ijk }}{\Sigma} \sum \mathrm{p}_{\mathrm{ijk}}=1.1
$$

Tables formed by summing $p_{i j k}$ over all categories of one or more variables are called marginal tables. The variables summed over will be represented by dots; thus, the entries in the AB marginal table would be the values of $p_{i j}$, , those in the $B$ marginal table the values of $p_{\cdot j} \cdot$, etc. The observed frequency in the cell characterized by level $i$ of $A, j$ of $B$, and $k$ of $C$ will be denoted $N_{i j k}$, with the total frequency,

$$
\underset{i j k}{\sum \sum N_{i j k}}
$$

denoted by $\mathbf{N}$. The dot notation used for probabilities will be used similarly for observed frequencies; thus

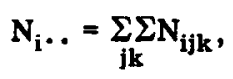

etc. Any term enclosed in parentheses will refer to the set of such values in the entire table; e.g., $\left(\mathbf{N}_{i \cdot k}\right)$ denotes the complete set of marginal AC frequencies.

\section{THE LOG-LINEAR MODEL}

The log-linear model for a three-variable contingency table can be written in the form:

$$
\begin{aligned}
\log p_{i j k}=v+v_{1 i}+v_{2 j}+v_{3 k} & +v_{12 i j}+v_{13 i k} \\
& +v_{23 j k}+v_{123 i j k}
\end{aligned}
$$

$\mathrm{i}=1, \cdots, \mathrm{a} ; \mathrm{j}=1, \cdots, \mathrm{b} ; \mathrm{k}=1, \cdots, \mathrm{c}^{2}$ The exact correspondence between the contingency table model and a three-factor factorial analysis of variance model is apparent, and extends to the restrictions on the parameters; i.e.,

$$
\underset{i}{\sum v_{1 i}}=\sum_{j} v_{2 j}=\sum_{k} v_{3 k}=\sum_{i} v_{12 i j}=\sum_{i} v_{12 i j}=0
$$

etc. The models for higher-order contingency tables similarly correspond exactly to higher-order analysis of variance models. Hypotheses are formulated as in the corresponding analysis of variance models; e.g., for a three-factor table, the hypothesis of zero $\mathrm{A} \times \mathrm{B} \times \mathrm{C}$ interaction would be formulated as $v_{123 i j k}=0, i=1, \cdots$, $a ; j=1, \cdots, b ; k=1, \cdots, c$; the hypothesis of zero $\mathbf{A} \times \mathbf{B}$ 
interaction would be formulated as $v_{12 i j}=0, i=1, \cdots, a ; j$ $=1, \cdots, b$; etc. The meanings of the contingency table hypotheses are, however, different from the meanings of their formally equivalent counterparts in analysis of variance. In particular, the equivalents of main effects, single variable effects, would generally be of little interest in a contingency table context. (See Shaffer, 1972.)

\section{FIXED AND RANDOM \\ MARGINAL FREQUENCIES}

In obtaining contingency table data, all marginal tables may contain randomly sampled frequencies, or some or all marginal tables may be fixed. For example, a three-factor table where $A=\operatorname{sex}, B=$ race, and $C=$ hair color could be obtained in a variety of ways, such as (1) taking a random sample of individuals and classifying them according to the three criteria; (2) taking an equal number of males and females and classifying on the basis of the other criteria; or (3) taking equal numbers of white males, white females, black males, black females, etc., and classifying them on the basis of hair color. The three examples given would correspond to the conditions (1) no marginal frequencies fixed; (2) A marginal frequencies fixed, i.e., fixed $\left(\mathbf{N}_{\mathbf{i}} ..\right)$; and (3) AB marginal frequencies fixed, i.e., fixed $\left(\mathbf{N}_{\mathbf{i j}} \cdot\right)$. Note that if a marginal table contains fixed frequencies, all lower-order marginal tables that can be obtained from that table also contain fixed frequencies; e.g., in (3), the fixed AB marginal frequencies imply fixed A marginal frequencies, $\left(\mathrm{N}_{\mathrm{i}} \ldots\right)$, and fixed $\mathrm{B}$ marginal frequencies, (N.j.).

The methods developed here are appropriate for testing hypotheses on only those effects which do not correspond to fixed marginal frequencies. In (1) above, for instance, hypotheses corresponding to all effects can be tested; in (2), all hypotheses can be tested except that representing the main effect of $A$ (i.e., the hypothesis $v_{1 i}=0$ could not be tested); and in ( 3 ), the hypotheses which cannot be tested using this approach are: $v_{12 i j}=0, v_{1 i}=0$, and $v_{2 j}=$ 0 .

\section{HIERARCHICAL AND}

\section{NONHIERARCHICAL HYPOTHESES}

In a contingency table, the hypothesis that each effect in the general model is equal to zero can be tested individually, as is usually done in analysis of variance. For example, in a three-factor table, the hypothesis $\mathbf{A} \times \mathbf{B}=0$ would be expressed $v_{12 i j}=0$ for all $i$ and $j$; the hypothesis $A \times C=0$ would be expressed $v_{13 i k}=0$ for all $i$ and $k$; etc. These will be called nonhierarchical hypotheses. On the other hand, hypotheses may be of a form more frequently encountered in regresion analysis; for example, the hypotheris that $A$ does not interact with any of the other variables would be expressed in the form $v_{12 i j}=v_{13 i k}=$ $v_{123 i j k}=0$. The hypothesis that there is no A X B interaction could be taken to imply no $A \times B$ interaction at any level of $C$, and thus that both $v_{12 i j}$ and $v_{123 i j k}$ equal zero. Hypotheses of this latter kind, where the hypothesis that a particular effect equals zero implies that all higher-order interactions including the same set of variables equal zero, will be referred to as hierarchical hypotheses. As another example, the hypothesis that all frequencies in a three-factor contingency table can be accounted for by main effects of the factors, i.e., that the factors are all mutually independent, can be expressed as the set of hierarchical hypotheses $A \times B=0, A \times C=0$, and $B \times C=0$ (since, as hierarchical hypotheses, they imply $A^{-} \times B \times C=0$ ). A limited use of hierarchical hypotheses in analysis of variance has been proposed by Anderson (1968). Note that, under the definitions given, the hypothesis that the highest-order interaction equals zero is the only one which can be considered both bierarchical and nonhierarchical. It will be more convenient to consider it a a hierarchieal hypothesis.
Since the exact test procedure for a hierarchical hypothesis is much easier to understand than the procedure for testing a nonhierarchical hypothesis, the hierarchical procedure will be derived and illustrated first. For simplicity, the exact tests will be derived by considering the distribution appropriate to the situation where all variables are sampled randomly. However, as in the case of Fisher's exact test, the tests derived are equally appropriate if some of the variables have fixed marginal distributions.

\section{HIERARCHICAL HYPOTHESES The Three-Factor Table: \\ $\mathrm{A} \times \mathrm{B} \times \mathbf{C}=\mathbf{0}$}

Given a fixed total number of observations, $N$, the exact probability of obtaining any particular configuration of cell frequencies $\left(\mathrm{N}_{\mathrm{ijk}}\right)$ is given by the multinomial distribution:

$$
\text { Prob }\left[\left(N_{i j k}\right)\right]=\frac{N !}{\prod_{i, j, k} N_{i j k} !} \prod_{i, j, k}\left(p_{i j k} N_{i j k}\right) \text {. }
$$

Expressing $\log p_{i j k}$ as in Eq. 1, setting $v_{123 i j k}=0$ as implied by the hypothesis, and taking antilogarithms, yields

$$
p_{i j k}=e^{\left(v+v_{1 i}+v_{2 j}+v_{3 k}+v_{12 j j}+v_{13 i k}+v_{23 j k}\right)}
$$

Letting

$$
\begin{aligned}
& a_{1 i j}=e^{\left(v+v_{1 i}+v_{2 j}+v_{12 i j}\right)}, \\
& a_{2 i k}=e^{\left(v_{3 k}+v_{13 i k}\right)},
\end{aligned}
$$

and

$$
a_{3 j k}=e^{\left(v_{23 j k}\right)},
$$

the cell probabilities can be expressed

$$
p_{\mathbf{i j k}}=a_{1 \mathbf{j}} \mathrm{a}_{2 \mathbf{2}} \mathrm{a}_{\mathbf{3 j k}}
$$

for all $i, j$, and $k^{3}$ Substituting the expression for $p_{i j k}$ in Eq. 4 into Eq. 2 and simplifying yields

$$
\begin{aligned}
& \text { Prob }\left[\left(N_{i j k}\right) \mid A \times B \times C=0\right] \\
& =\frac{N !}{\prod_{i, j, k} N_{i j k} !} \prod_{i, j} a_{1 j j} N_{i j} \cdot \prod_{i, k} a_{2 i k} N_{i \cdot k} \prod_{i, k} a_{3 j k} N_{\cdot j k}
\end{aligned}
$$

Note that the portion of the product in Eq. 5 involving the unknown parameters $\left(a_{1 j}\right),\left(a_{2 i k}\right)$, and $\left(a_{3 j k}\right)$ is a function of the two-factor marginal frequencies only. It follows, then, that this portion is identical for all three-factor tables with identical two-factor marginal frequencies.

The probability of obtaining a specific set of cell frequencies, conditional on the obtained two-factor marginal frequencies, is defined as:

$$
\begin{aligned}
\operatorname{Prob}\left[\left(N_{i j k}\right) /\left(N_{i j \cdot}\right),\left(N_{i \cdot k}\right),\left(N_{\cdot j k}\right)\right] \\
\left.\left.=\frac{\frac{N !}{\prod_{i, j, k} N_{i j k} !} \prod_{i, j, k}\left(p_{i j k} N_{i j k}\right)}{\sum\left[\frac { N ! } { \prod _ { i , j , k } N _ { i j k } ^ { \prime } ! } \prod _ { i , j , k } \left(p_{i j k} N^{\prime} i j k\right.\right.}\right)\right]
\end{aligned}
$$

where the sum is taken over all sets $\left(N^{\prime}{ }_{i j k}\right)$ such that $\left(N_{i j \cdot}\right)$ $=\left(N_{i j \cdot}\right),\left(N_{i \cdot k}^{\prime}\right)=\left(N_{i \cdot k}\right)$, and $\left.\left(N^{\prime} \cdot j k\right)=N_{\cdot j k}\right)$. If $A \times B \times C=$ 0 , the expresion in $\mathrm{Eq}$. 5 can be substituted into both the numerator and denominator of Eq. 6. Simplifying, the 
Table 1

Tables of Frequencies Required for the Exact Test of $A \times B \times C=0$ in the Given 2 by 2 by 3 Contingency Table

\begin{tabular}{cccc} 
& $C=1$ & $C=2$ & $C=3$ \\
\cline { 3 - 4 } & $B=1 \quad B=2$ & $B=1 \quad B=2$ & $B=1 \quad B=2$ \\
\hline
\end{tabular}

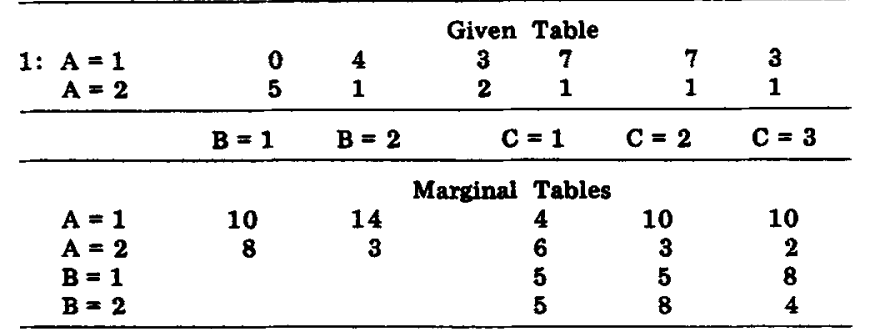

$\begin{array}{ccc}C=1 & C=2 & C=3 \\ B=1 B=2 & B=1 . B=2 & B=1 . B=2\end{array}$

\begin{tabular}{rlrllll} 
& \multicolumn{7}{c}{ Other Tables with Same Marginal Frequencies } \\
2: $\mathbf{A}=\mathbf{1}$ & $\mathbf{1}$ & $\mathbf{3}$ & $\mathbf{4}$ & $\mathbf{6}$ & $\mathbf{6}$ & $\mathbf{4}$ \\
$\mathbf{A}=\mathbf{2}$ & $\mathbf{4}$ & $\mathbf{2}$ & $\mathbf{1}$ & $\mathbf{2}$ & $\mathbf{2}$ & $\mathbf{0}$ \\
3: $\mathbf{A}=\mathbf{1}$ & $\mathbf{0}$ & 4 & $\mathbf{2}$ & $\mathbf{8}$ & $\mathbf{8}$ & $\mathbf{2}$ \\
$\mathbf{A}=\mathbf{2}$ & $\mathbf{5}$ & $\mathbf{1}$ & $\mathbf{3}$ & $\mathbf{0}$ & $\mathbf{0}$ & $\mathbf{2}$ \\
4: $\mathbf{A}=\mathbf{1}$ & $\mathbf{1}$ & $\mathbf{3}$ & $\mathbf{3}$ & $\mathbf{7}$ & 6 & $\mathbf{4}$ \\
$\mathbf{A}=\mathbf{2}$ & $\mathbf{4}$ & $\mathbf{2}$ & $\mathbf{2}$ & $\mathbf{1}$ & $\mathbf{2}$ & $\mathbf{0}$ \\
5: $\mathbf{A}=\mathbf{1}$ & $\mathbf{1}$ & $\mathbf{3}$ & $\mathbf{2}$ & $\mathbf{8}$ & $\mathbf{7}$ & $\mathbf{3}$ \\
$\mathbf{A}=\mathbf{2}$ & $\mathbf{4}$ & $\mathbf{2}$ & $\mathbf{3}$ & $\mathbf{0}$ & $\mathbf{1}$ & $\mathbf{1}$ \\
$\mathbf{6}=\mathbf{1}$ & $\mathbf{2}$ & $\mathbf{2}$ & $\mathbf{2}$ & $\mathbf{8}$ & 6 & $\mathbf{4}$ \\
$\mathbf{A}=\mathbf{2}$ & $\mathbf{3}$ & $\mathbf{3}$ & $\mathbf{3}$ & $\mathbf{0}$ & $\mathbf{2}$ & $\mathbf{0}$ \\
\hline
\end{tabular}

conditional probability of obtaining the set of frequencies $\left(\mathrm{N}_{\mathrm{ijk}}\right)$, given $\mathrm{A} \times \mathrm{B} \times \mathrm{C}=0$ and the fixed two-factor marginal frequencies, can be expressed:

$$
\begin{array}{r}
\text { Prob }\left[\left(\mathrm{N}_{\mathrm{ijk}}\right) \mid A \times B \times C=0 \text { and }\left(\mathrm{N}_{\mathrm{ij} \cdot}\right),\left(\mathrm{N}_{\mathrm{i} \cdot \mathrm{k}}\right),\left(\mathrm{N}_{\cdot \mathrm{jk}}\right)\right] \\
=\frac{\frac{1}{\prod_{\mathrm{i}, \mathrm{k}, \mathrm{k}} \mathrm{N}_{\mathrm{ijk}} !}}{\sum\left(\frac{1}{\prod_{\mathrm{i}, \mathrm{j}, \mathrm{k}} \mathrm{N}_{\mathrm{ijk}}^{\prime} !}\right)}
\end{array}
$$

where the sum is taken over all sets of frequencies $\left(N_{i j k}\right)$ satisfying the marginal restrictions as enumerated above. The expression in Eq. 7 involves no unknown parameters and can be computed exactly. Its use in developing an exact test is analogous to the use of the expression for the exact probability of the obtained frequencies in a $2 \times 2$ table in applying Fisher's exact test; the latter probability is conditional on fixed one-factor marginal frequencies. In the three-factor case, no single closed expression for the sum in the denominator can be obtained as it can in the two-factor case.

As in the case of Fisher's exact test, it is not sufficient to determine the exact probability of the obtained table. All possible tables of frequencies which would satisfy the marginal restrictions must be considered, and exact probabilities are needed for those tables in which frequencies deviate from expectation as much as or more than in the obtained table. The sum of the probabilities of all such tables is the exact probability level with respect to the test of the hypothesis. The smaller the exact probability of a table, the more deviant from expectation it is considered. The subset of all possible tables with exact probability smaller than or equal to that of the obtained table is determined. The sum of the probabilities of all tables in this subset, which includes the obtained table, is the exact probability relevant to the test of the hypothesis. If this probability is smaller than or equal to an acceptable significance level, e.g., .05 , the hypothesis $\mathrm{A} \times \mathrm{B} \times \mathrm{C}=0$ can be rejected. Althoug: the test is conditional on the marginal frequencies, note that, for any set of marginal frequencies, the null hypothesis would be rejected if, and only if, the exact $p$ values were smaller than or equal to the level of significance adopted. Therefore, the overall unconditional probability of rejection is smaller than or equal to the significance level chosen. The reasoning in this respect is the same as that justifying Fisher's exact test (Mood \& Graybill, 1963). When some of the variables are not randomly sampled but have fixed marginal frequencies, the initial exact probability reflects these fixed frequencies, and the final test statistic is identical.

As an illustration, the method will be applied to test the significance of $A \times B \times C$ interaction in a $2 \times 2 \times 3$ table. Table 1 gives the frequencies in the contingency table, followed by the three two-factor marginal tables derived from it. Below these are listed the other possible tables of frequencies, five in all, which have the same marginal tables. The probability computations are simpler with the use of a table of logarithms of factorials; these are readily available. Table 2 illustrates the computations in detail. The logarithm of the numerator of the exact probability for each table, from Eq. 7, equals

$$
-\sum \sum \sum \log \mathbf{N}_{\mathbf{i j k}} !_{\mathbf{j}}
$$

These values are listed for each table. It is convenient to add

$$
\sum \sum_{\mathrm{ijk}} \sum \log \mathrm{N}_{\mathrm{ijk}} \text { ! }
$$

to each value before taking antilogarithms; this results in more manageable numbers but does not affect the final probability. The resulting value for the obtained table is then necessarily zero, with antilogarithm equal to one; antilogarithms are listed for each table. The required probability is the sum of the antilogarithms which are less than or equal to one divided by the sum of all the antilogarithms, or 0.07 in this example, so that the $\mathrm{A} \times \mathrm{B} \times \mathrm{C}$ interaction is of borderline significance.

$$
\begin{aligned}
& \text { The Three-Factor Table: } \\
& \text { A x B }=0
\end{aligned}
$$

The hierarchical hypothesis $\mathrm{A} \times \mathrm{B}=0$ implies the two individual hypotheses $\mathrm{A} \times \mathrm{B}=0$ and $\mathrm{A} \times \mathrm{B} \times \mathrm{C}=0$. Using the formula for $\log p_{\mathrm{ijk}}$ (Eq. 1), setting $v_{12 \mathrm{ij}}$ and $\mathrm{v}_{123 \mathrm{ijk}}=$ 0 , taking the antilogarithm, and decomposing the exponent, yields

$$
p_{i j k}=a_{4 i k} a_{5 j k}
$$

where

$$
a_{4 i k}=e^{\left(v+v_{1 i}+v_{3 k}+v_{13 i k}\right)} \text { and } a_{5 j k}=e^{\left(v_{2 j}+v_{23 j k}\right)}
$$

Table 2

Calculations for Exact Test of Hypothesis $A \times B \times C=0$ from Data in Table 1

\begin{tabular}{cccl}
\hline Table & $\begin{array}{c}-\sum \sum \sum \log _{10} \mathrm{~N}^{\prime} \mathrm{jik} ! \\
\text { ijk }\end{array}$ & $\begin{array}{c}-\sum \Sigma \sum \log _{10} \mathrm{~N}^{\prime} \mathrm{ijk} ! \\
+12.722\end{array}$ & Antilog \\
\hline 1 & -12.722 & 0 & 1.00 \\
2 & -12.537 & 0.185 & 1.53 \\
3 & -14.352 & -1.630 & 0.023 \\
4 & -11.780 & 0.942 & $\mathbf{8 . 7 5}$ \\
5 & -12.625 & 0.097 & 1.25 \\
6 & -12.382 & 0.340 & 2.19 \\
\hline
\end{tabular}

Note: $p=\frac{1.023}{14.74}=.07$ 
for all $i, j$, and $k$. Substituting the expression for $p_{i j k}$ in Eq. 8 into Eq. 2 and simplifying yields

$$
\begin{aligned}
& \text { Prob }\left[\left(N_{i j k}\right) \mid A \times B=0 \text { and } A \times B \times C=0\right] \\
& =\frac{N !}{\prod_{i, j, k} N_{i j k} !} \prod_{i, k}\left(a_{4 i k} N_{i \cdot k}\right) \prod_{j, k}\left(a_{5 j k}{ }^{N} \cdot j k\right)
\end{aligned}
$$

and, dividing by the probability of obtaining a table with the same marginal $\mathrm{AC}$ and marginal $\mathrm{BC}$ frequencies, and simplifying, the exact probability of the obtained table, conditional on the hypotheses and on the $\mathrm{AC}$ and $\mathrm{BC}$ marginal frequencies, is

$$
\text { Prob }\left[\left(\mathrm{N}_{\mathrm{jjk}}\right) \mid A \times B=0, A \times B \times C=0,\left(\mathrm{~N}_{\mathrm{i} \cdot \mathrm{k}}\right),\left(\mathrm{N}_{\cdot \mathrm{jk}}\right)\right]
$$

$$
=\frac{\frac{1}{\prod_{i, j, k} N_{i j k} !}}{\sum\left(\frac{1}{\prod_{i, j, k} N^{\prime}{ }_{i j k} !}\right)}
$$

where the sum is taken over all sets of $\left(\mathrm{N}^{\prime}{ }_{\mathrm{ijk}}\right)$ such that $\left(N_{i \cdot k}^{\prime}\right)=\left(N_{i \cdot k}\right)$ and $\left(N^{\prime} \cdot j k\right)=\left(N_{\cdot j k}\right)$. Proceeding in the same manner as for the previous exact test, an exact joint test of the hypotheses $\mathrm{A} \times \mathrm{B}=0$ and $\mathrm{A} \times \mathrm{B} \times \mathrm{C}=0$ is obtained.

As an example, this test was performed on the contingency table given in Table 1 . The number of possible tables satisfying the two marginal restrictions is much larger than the number satisfying the three marginal restrictions in testing $\mathrm{A} \times \mathrm{B} \times \mathrm{C}=0$. The procedure can be simplified considerably in the following way:

First, by using the formulation of the log-linear model, it can easily be shown that $a_{4 j k}=p_{i \cdot k}$ and $a_{5 j k}=p_{\cdot j k} / p \cdot \cdot_{k}$. Then Eq. 9 can be equivalently expressed in the form

Prob $\left[\left(\mathrm{N}_{\mathrm{ijk}}\right) \mid A \times B=0\right.$ and $\left.A \times B \times C=0\right]$

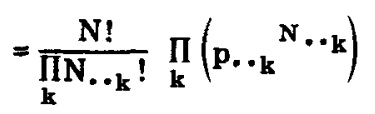

$$
\begin{aligned}
& \cdot \prod_{k}\left[\frac{N_{\cdot_{k}} !}{\prod_{i, j} N_{i j k} !} \prod_{i}\left(\frac{p_{i \cdot k}}{p_{\cdot_{k}}}\right)^{N_{i} \cdot k} \prod_{j}\left(\frac{p_{\cdot j k}}{p_{\cdot \cdot k}}\right)^{N} \cdot\right.
\end{aligned}
$$

If the $\mathrm{AC}$ and $\mathrm{BC}$ marginal frequencies are to be fixed, then the $C$ marginal frequencies must necessarily be fixed. The probability conditional on the fixed $\mathbf{C}$ marginal frequencies, $\left(\mathrm{N} . ._{\mathbf{k}}\right)$, is

Prob $\left[\left(\mathrm{N}_{\mathrm{ijk}}\right) \mid A \times B=0, A \times B \times C=0\right.$, and $\left.\left(\mathrm{N}_{\cdot \cdot k}\right)\right]$

$$
=\frac{\text { Prob }\left[\left(\mathrm{N}_{\mathrm{ijk}}\right) \mid A \times B=0, A \times B \times C=0\right]}{\text { Prob }\left[\left(\mathrm{N}_{. .}\right) \mid A \times B=0, A \times B \times C=0\right]} .
$$

The denominator of Eq. 12 is

Prob $\left[\left(\mathrm{N}_{. .}\right) \mid \mathrm{A} \times \mathrm{B}=0, \mathrm{~A} \times \mathrm{B} \times \mathrm{C}=0\right]$

$$
=\frac{N !}{\prod_{k} N \cdot_{k} !} \prod_{k} p_{\cdot \cdot k}{ }^{N} \cdot \cdot_{k}
$$

Dividing Eq. 11 by Eq. 13 yields
Prob $\left[\left(\mathrm{N}_{\mathrm{ijk}}\right) \mid \mathrm{A} \times \mathrm{B}=0, \mathrm{~A} \times \mathrm{B} \times \mathrm{C}=0\right.$, and $\left.\left(\mathrm{N}_{. \cdot \mathrm{k}}\right)\right]$

$$
\begin{aligned}
& =\prod_{k}\left(\frac{N_{\cdot \cdot k} !}{\prod_{i, j} N_{i j k} !}\right) \prod_{i}\left(\frac{p_{i \cdot k}}{p_{\cdot \cdot k}}\right)^{N_{i \cdot k}} \prod_{j}\left(\frac{p_{\cdot j k}}{p_{\cdot \cdot k}}\right)^{N_{\cdot j k}} \\
& =\prod_{k} \operatorname{Prob}\left[\left(N_{i j}\right)_{k} \mid k, N . . . k_{k}\right]
\end{aligned}
$$

where $\left(\mathrm{N}_{\mathrm{ij}}\right)_{\mathrm{k}}$ refers to the set of cell frequencies for a given value of $k$ and all $i, j$.

In words, the foregoing development shows that the probability of each table, given $\left(\mathrm{N} \cdot ._{k}\right)$, can be evaluated by finding the probability of each two-factor $A B$ table for fixed $k$, and getting the product of these probabilities. Evaluating the probability of each two-factor table conditional on fixed $\left(\mathrm{N}_{\mathrm{i} \cdot \mathrm{k}}\right)$ and $\left(\mathrm{N}_{\cdot \mathrm{jk}}\right)$ is equivalent to evaluating the probability of a two-dimensional table with fixed one-dimensional margins. This probability can be expressed in closed form (see Mood \& Graybill, 1963, p. 316), yielding

$$
\begin{aligned}
& \operatorname{Prob}\left[\left(N_{i j k}\right) \mid A \times B\right.\left.=0, A \times B \times C=0,\left(N_{i \cdot k}\right),\left(N_{\cdot j k}\right)\right] \\
&=\prod_{k}\left(\frac{\prod_{i} N_{i \cdot k} ! \prod_{i} N_{\cdot j k} !}{N . \cdot_{k} ! \prod_{i, j} N_{i j k} !}\right)
\end{aligned}
$$

As applied to the test of the hypotheses $\mathrm{A} \times \mathrm{B}=0$ and $\mathrm{A} \times \mathrm{B} \times \mathrm{C}=0$ in the $2 \times 2 \times 3$ contingency table given in Table 1, note that the table consists of three $2 \times 2$ tables, one for each value of $k$. There are five $2 \times 2$ tables satisfying the marginal restrictions for $k=1$, four for $k=2$, and three for $k=3$. The total number of $2 \times 2 \times 3$ tables satisfying the marginal restrictions is therefore $5 \times 4 \times 3=$ 60. The probability of each total table is the product of the probabilities of each of the three $2 \times 2$ tables composing it. The sum of the probabilities of the obtained table and all tables less or equally probable equals .03 , which is therefore the exact probability associated with the test of the hypothesis. Thus, the hypothesis can be rejected at the $3 \%$ level.

Fortunately, it seems likely that whenever the number of alternative tables is as large as in this example, the chi-square approximate test (Shaffer, 1972) is accurate enough for all practical purposes. In this example, $x^{2}=8.28$, df $=3, p=.04$.

The same general principles can be used to simplify the determination of alternative contingency tables and compute their probabilities under other hierarchical hypotheses. For instance, in a three-factor table, if the hypothesis is $\mathrm{A} \times \mathrm{B}=0, \mathrm{~A} \times \mathrm{C}=0, \mathrm{~A} \times \mathrm{B} \times \mathrm{C}=0$, the probability of each table is

Prob $\left[\left(N_{i j k}\right) \mid A \times B=0, A \times C=0\right.$,

$$
\left.A \times B \times C=0,\left(N_{i}, \ldots\right),\left(N_{\cdot j k}\right)\right]
$$

$$
=\frac{\prod_{i} N_{i} . . ! \prod_{j, k} N_{\cdot j k} !}{N ! \prod_{i, j, k} N_{i j k} !} .
$$

If the hypothesis is $\mathrm{A} \times \mathrm{B}=0, \mathrm{~A} \times \mathrm{C}=0, \mathrm{~B} \times \mathrm{C}=0$, $A \times B \times C=0$, i.e., complete independence of all factors, the conditional probability of each table is 
Prob $\left[\left(\mathrm{N}_{\mathrm{ijk}}\right) \mid A \times B=0, A \times C=0, B \times C=0\right.$,

$$
\begin{aligned}
\left.A \times B \times C=0,\left(N_{i} \cdot .\right),\left(N_{\cdot j} \cdot\right),\left(N ._{k}\right)\right] \\
=\frac{\prod_{i} N_{i}, . ! \prod_{j} N_{\cdot j} ! \prod_{k} N ._{k} !}{(N !)^{2} \prod_{i, j, k} N_{i j k} !}
\end{aligned}
$$

\section{Exact Tests of Hierarchical Hypotheses}

in the General Contingency Table

The general approach can now be simply stated. In a hierarchical system, the hypothesis that a given effect is zero implies that all interactions involving the same combination of variables are zero. The set of effects assumed to be zero should be written out. The marginal tables involving all other combinations of variables should be considered fixed. The exact probability of obtaining the given sample, conditional on the hypotheses and on these fixed marginal frequencies, is

Prob $\left[\left(\mathbf{N}_{\mathrm{ijk}}\right) \mid\right.$ hypotheses and marginal restrictions ]

$$
=\frac{\frac{1}{\prod_{i, j, k} N_{i j k} !}}{\sum\left(\frac{1}{\prod_{i, j, k} N_{i j k}^{\prime} !}\right)}
$$

where the summation is taken over all sets of values $\left(\mathrm{N}^{\prime}{ }_{i j k}\right)$ such that the fixed marginal values are preserved. The sum of the probability of the obtained set of frequencies and all less probable sets of frequencies is determined. If this sum is less than or equal to an acceptable significance level, the set of null hypotheses can be rejected.

For example, suppose, in a four-factor table, a test of the pair of hierarchical hypotheses $A \times B=0$ and $B \times C \times D=0$ is desired. The full set of hypotheses is then $A \times B=0$, $A \times B \times C=0, A \times B \times D=0, A \times B \times C \times D=0, B \times C \times D$ $=0$. The marginal tables to be fixed can be obtained as follows: the full set of effects is written out and all those assumed to be zero are eliminated (indicate by line drawn through). All marginal totals which are automatically fixed as a consequence of fixing other marginal tables are enclosed in parentheses. (For example, if the ACD marginal totals are fixed, the $A C, A D, C D, A, C$, and D marginal frequencies are automatically fixed as a result.) The remaining marginal frequencies (circle) constitute the set which must be kept fixed when obtaining alternative sets of frequencies in order to carry out an exact test. For this example, the list would look as follows:

$$
\begin{aligned}
& \text { (A), (B), (C), (D), A\#, (AC), (AD), BC , BD, } \\
& \text { (CD), ABG, } A B D, \text { ACD, } B G B, A B G P \text {. }
\end{aligned}
$$$$
\text { NONHIERARCHICAL HYPOTHESES }
$$

The extension of the exact approach to deal with nonhierarchical hypotheses will be given in detail in the special case of a test of two-factor interaction in a three-factor table; the application to the general case will then be described.

\section{The Exact Test in the \\ Three-Factor Table:}

$$
\text { A } \times \text { B }=0
$$

In order to perform the exact test, it is necessary to find a set of conditions such that the probability of all tables fulfilling those conditions can be computed exactly, independently of the unknown parameters. In the case of hierarchical hypotheses, the conditions can always be expressed in the form of restrictions on sets of marginal frequencies, i.e., frequencies in specified marginal tables. With nonhierarchical hypotheses, the conditions are more complex. They can be most conveniently derived from a combination of Eqs. 1 and 2 obtained by substituting the general expression for $p_{\mathrm{ijk}}$ implied by Eq. 1 into Eq. 2 and simplifying, yielding

$$
\begin{aligned}
& \text { Prob }\left[\left(N_{i j k}\right)\right]=\frac{N !}{\prod_{i, j, k} N_{i j k} !} \exp \left(v N+\sum_{i} v_{1 i} N_{i} . .+\sum_{j} v_{2 j} N_{\cdot j} \cdot\right. \\
& +\sum_{k} \mathbf{v}_{3 k} N_{\cdot \cdot k}+\sum_{i j} \sum v_{12 i j} N_{i j} \cdot+\sum_{i k} \sum_{13 i k} N_{i \cdot k} \\
& \left.+\sum_{j k} \sum v_{23 j k} N_{\cdot j k}+\sum_{i j k} \sum v_{123 i j k} N_{i j k}\right)
\end{aligned}
$$

The hypothesis $\mathrm{A} \times \mathrm{x} \mathrm{B}=\mathbf{0}$ can be expressed equivalently as $v_{12}$ ij $=0$, for all $i$ and $j$. The exponent of $e$ in Eq. 19 would be identical for all tables with the same $A C$ and $B C$ marginal frequencies, and $\left(\mathrm{N}^{\prime}{ }_{\mathrm{ijk}}\right)$ for which

$$
\underset{\mathrm{ijk}}{\sum \sum \mathbf{v}_{123 \mathrm{ijk}}} \mathrm{N}_{\mathrm{ijk}}=\sum \sum_{\mathrm{ijk}} \sum \mathrm{v}_{123 \mathrm{ijk}} \mathrm{N}_{\mathrm{ijk}} .
$$

The latter can be expressed in terms of relationships among the cell frequencies by use of the restrictions on $v_{123 \mathrm{ijk}}$ :

$$
\sum_{i} v_{123 i j k}=\sum_{j} v_{123 i j k}=\sum_{k} v_{123 i j k}=0 .
$$

For a $2^{3}$ table, for example, these restrictions are equivalent to the requirements that $v_{123111}=v_{123122}=$ $v_{123212}=v_{123221}=-v_{123112}=-v_{123121}=-v_{123211}$ $=-v_{123222}$, and therefore

$$
\begin{aligned}
& \sum_{\mathrm{ijk}} \sum \mathrm{v}_{123 \mathrm{ijk}} \mathrm{N}_{\mathrm{ijk}} \\
& =\mathrm{v}_{123111}\left(\mathrm{~N}_{111}+\mathrm{N}_{122}+\mathrm{N}_{212}+\mathrm{N}_{221}-\mathrm{N}_{112}\right. \\
& \left.-\mathrm{N}_{121}-\mathrm{N}_{211}-\mathrm{N}_{222}\right) .
\end{aligned}
$$

It follows that in testing the hypothesis $\mathrm{A} \times \mathrm{B}=0$ in a $2^{3}$ table, the probability of the observed set of frequencies $\left(N_{i j k}\right)$ should be taken conditional on tables with frequencies $\left(N^{\prime}{ }_{i j k}\right)$ satisfying the conditions, for all $i, j$, and $\mathrm{k}:$ (a) $\mathrm{N}_{\mathrm{i} \cdot \mathrm{k}}^{\prime}=\mathrm{N}_{\mathrm{i} \cdot \mathrm{k}}$, (b) $\mathrm{N}^{\prime} \cdot \mathrm{jk}_{\mathrm{k}}=\mathrm{N}_{\cdot \mathrm{jk}}$, and (c) $\mathrm{N}^{\prime}{ }_{111}+$ $\mathrm{N}_{122}+\mathrm{N}_{212}+\mathrm{N}_{221}-\mathrm{N}_{112}-\mathrm{N}_{121}^{\prime}-\mathrm{N}_{211}^{\prime}$ $\mathrm{N}_{222}^{\prime}=\mathrm{N}_{111}+\mathrm{N}_{122}+\mathrm{N}_{212}+\mathrm{N}_{221}-\mathrm{N}_{112}-\mathrm{N}_{121}-$ $\mathrm{N}_{211}-\mathrm{N}_{222}$. The exact test consists of listing all such tables, computing

$$
-\sum \sum_{\mathrm{ijk}} \sum \log \mathrm{N}_{\mathrm{ijk}}^{\prime}
$$

for each, and proceeding as in the exact tests of hierarchical hypotheses.

For general three-factor tables, the only modification of the above specifications is that Condition $c$ must be satisfied for each $2^{3}$ subtable. Since it is sufficient to specify a subset of all subtables, for example, those consisting of adjacent index values on each variable (see Shaffer, 1972), Condition c for a general three-factor table can be expressed: $N^{\prime} N_{j k}+N_{i(j+1)(k+1)}+N^{\prime}(i+1) j(k+1)+$ $N_{N^{\prime}}^{\prime}(\mathbf{i}+1)(j+1) k-N_{i j(k+1)}-N_{i(j+1) k}-N_{(i+1) j k}^{\prime}-$ $N^{\prime}(i+1)(j+1)(k+1)=N_{i j k}+N_{i(j+1)(k+1)}+N_{(i+1) j(k+1)}+$ $N_{(i+1)(j+1) k}-N_{i j(k+1)}-N_{i(j+1) k}-N_{(i+1) j k}-$ $N_{(i+1)(j+1)(k+1)}$, for $i=1, \cdots, a-1 ; j=1, \cdots, b-1 ; k=$ $1, \cdots, c-1$.

As an illustration of the technique, the nonhierarchical 
hypothesis $\mathrm{A} \times \mathrm{B}=0$ is tested, using the contingency table for which the hypothesis $\mathrm{A} \times \mathrm{B} \times \mathrm{C}=0$ and the hierarchical hypothesis $A \times B=0$ were tested (see Tables 1 and 2). The marginal frequencies required for Conditions $a$ and $b$ are given in Table 1. Condition $c$ for this table can be expressed: $\mathrm{N}_{111}^{\prime}+\mathrm{N}_{122}^{\prime}+\mathrm{N}_{212}^{\prime}+\mathrm{N}_{221}^{\prime}-\mathrm{N}_{112}^{\prime}-$ $N_{121}^{\prime}-N_{211}^{\prime}-N_{222}^{\prime}=-3$, and $N_{112}^{\prime}+N_{123}^{\prime}+N_{213}^{1}$ $+N_{222}^{\prime}-N_{113}^{\prime}-N_{122}^{\prime}-N^{\prime}{ }_{212}-N_{223}^{\prime}=-9$. Aside from the original table, only one other set of frequencies $\left(N_{i j k}^{\prime}\right)$ satisfies Conditions $a, b$, and $c$, the set $N_{111}^{\prime}=1$, $N_{112}^{\prime}=4, N_{113}^{\prime}=8, N_{121}^{\prime}=3, N_{122}^{\prime}=6, N_{123}^{\prime}{ }_{12}^{\prime}=2$, $N_{211}^{\prime}=4, N_{212}^{\prime}=1, N_{213}^{\prime}=0, N_{221}^{\prime}=2, N_{222}^{\prime}=2$, and $\hat{N}_{223}^{\prime}=2$. Proceeding in the same way as in Table 2 , the exact $p$ value is equal to $(1 / 4.28)=.23$.

The Exact Test of Any Hypothesis in the General Contingency Table

In constructing the test of any hypothesis, it is helpful to start with Eq. 19 and, setting the hypothesized term or terms equal to zero, find the minimum set of restrictions on cell frequencies which would yield sets of frequencies with identical values of the exponent, independent of the values of the parameters $v$. In every case, these restrictions will be in terms of marginal frequencies or in terms of functions analogous to that expressed in Condition $c$ as written for the general three-factor table, functions either of the cell frequencies directly or of frequencies in marginal tables. In obtaining the restrictions, it will be simpler to obtain each of them for a table with two levels of each subscript of $\mathrm{N}^{\prime}$ which has not been replaced by dots in the expression; it can then be generalized by replacing the subscripts of the $\mathrm{N}^{\prime}$ s by $\mathrm{i}$ or $(\mathrm{i}+1), \mathrm{j}$ or $(\mathrm{j}+1)$, etc., according to the positions of the subscripts.

\section{LARGE-SAMPLE APPROXIMATE TESTS}

In a case where the number of alternative tables of frequencies satisfying the marginal restrictions is sufficiently large, the approximate chi-square techniques presented in Shaffer (1972) are appropriate Comparisons $_{2}$ of the power of alternative approximations, and determination of sample size conditions under which they are sufficiently accurate, remain for future research. Note that computer programs can be relatively easily developed for the exact methods for small and moderate sample sizes.

\section{REFERENCES}

ANDERSON, N. H. Partial analysis of high-way factorial designs. Behavior Research Methods \& Instrumentation, 1968, 1, 2-7.

BARTLETT, M. S. Contingency table interactions. Journal of the Royal Statistical Society, Supplement, 1935, 2,-248-252.

FREEMAN, G. H., \& HALTON, H. H. Note on an exact treatment of contingency, goodness of fit and other problems significance. Biometrika, 1951, 38, 141-149.

MOOD, A. M., \& GRAYBILL, F. A. Introduction to the theory of statistics, (2nd ed.) New York: McGraw-Hill, 1963.

SHAFFER, J. P. Defining and testing hypotheses in multidimensional contingency tables. Psychological Bulletin 1972 , in press.

\section{NOTES}

1. Summations and products will always be over the full range of possible values of the variables. Therefore, for convenience, the range will not be indicated in the summation or product notation.

2. The logarithms can be taken to any base, but natural logarithms will be assumed because of their greater convenience when considering approximate test procedures.

3 . The constants are clearly not uniquely determined, since the decomposition could have been carried out in alternate ways. However, uniqueness is neither assumed nor required in the ensuing analysis. 